\title{
The life and death of star clusters
}

\author{
B. C. Whitmore \\ ${ }^{1}$ Space Telescope Science Institute, 3700 San Martin Dr., Baltimore, MD, 21218, USA \\ email: whitmore@stsci.edu
}

\begin{abstract}
It is generally believed that most stars are born in groups and clusters, rather than in the field. It has also been demonstrated that merging galaxies produce large numbers of young massive star clusters, sometimes called super star clusters. Hence, understanding what triggers the formation of these young massive clusters may provide important information about what triggers the formation of stars in general. In recent years it has become apparent that most clusters do not survive more than $\approx 10$ Myr (i.e., "infant mortality"). Hence, it is just as important to understand the disruption of star clusters as it is to understand their formation if we want to understand the demographics of both star clusters and field stars. This talk will first discuss what triggers star cluster formation in merging galaxies (primarily in the Antennae galaxies), will then demonstrate that most of the faint objects detected in the Antennae are clusters rather than individual stars (which shows that the initial mass function was a power law rather than a Gaussian), and will then outline a general framework designed to empirically fit observations of both star clusters and field stars in a wide variety of galaxies from mergers to quiescent spirals.
\end{abstract}

\section{Introduction}

Why are we talking about star clusters in a meeting about the formation of stars? The obvious answer is that most stars are formed in associations, groups and clusters, (e.g., Lada \& Lada, 2003). Hence, understanding what triggers the formation of star clusters may provide important clues for understanding the formation of stars in general. It is well known that star formation is enhanced in merging galaxies, so this is a good place to start. The "Antennae Galaxies" (NGC 4038/39; see Whitmore et al. 1999 for image) are the youngest and nearest galaxies in the Toomre sequence (Toomre 1977) of prototypical mergers. Hence, they may represent our best chance for understanding the formation of star clusters in interacting galaxies. While other galaxies will occasionally be mentioned in this review, the primary focus will be the Antennae.

\section{What triggers the formation of star clusters}

\subsection{Kinematic clues}

While the details of star formation are still obscure, one thing that everyone agrees on is that shocks are important (e.g., along spirals arms). In mergers, one popular model (e.g., Kumai et al. 1993) has been that high-speed cloud-cloud collisions with velocities $\approx 50-100 \mathrm{~km} \mathrm{~s}^{-1}$ are required. We have used STIS long-slit spectra in three positions angle of the Antennae to test this idea (Whitmore et al. 2005).

We find the velocity fields are remarkably quiescent (Figure 1). RMS dispersions are $\approx 10 \mathrm{~km} \mathrm{~s}^{-1}$, essentially the same as disks of spiral galaxies. This does not favor highspeed cloud-cloud collision models, but is consistent with models where a high pressure interstellar medium implodes GMCs without greatly altering their velocity distribution (e.g, Jog and Solomon 1992). This also supports the results of Zhang, Fall, \& Whitmore 


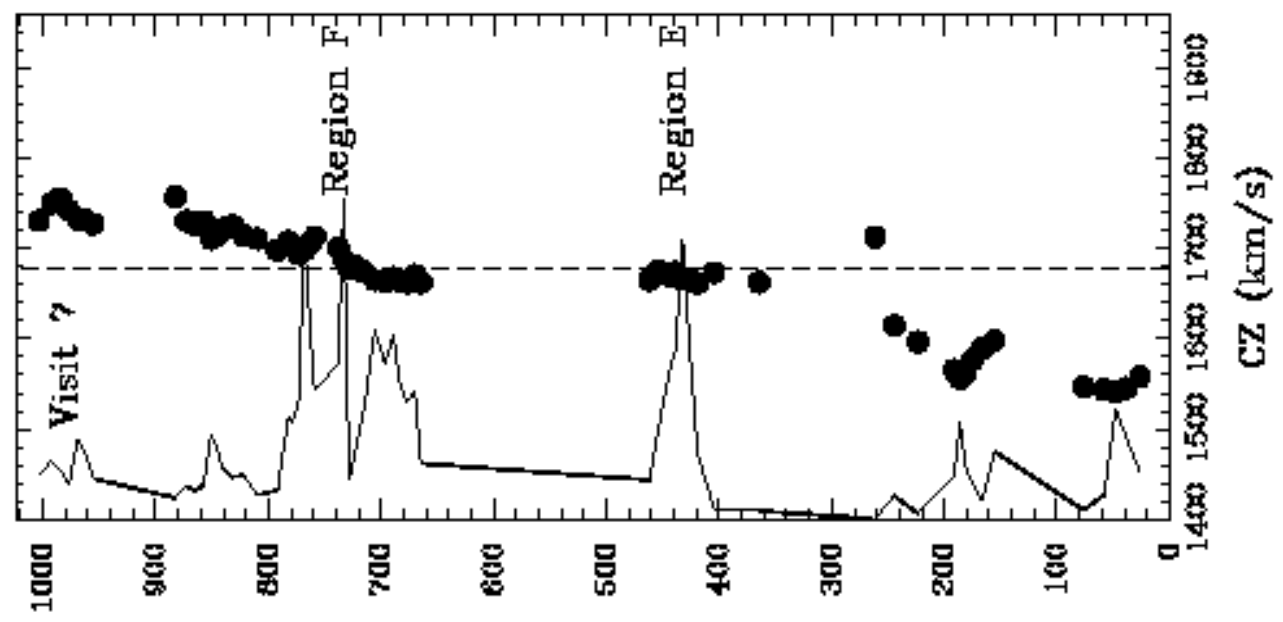

Figure 1. H $\alpha$ velocities of star clusters in the Antennae based on long-slit observations using the STIS detector on HST. Note how small the velocity dispersion is for a given subregion (e.g., $\approx 10 \mathrm{~km} \mathrm{~s}^{-1}$ for region $\mathrm{F}$, once the large scale gradient is removed). See Whitmore et al. 2005 for details.

(2001) who found essentially no correlation between the positions of young clusters in the Antennae and velocity gradients.

\subsection{Morphological clues}

Another approach to studying triggered star formation is to age-date the stars or star clusters and then look for patterns that suggest sequential star formation. One of the classic example of this is 30 Doradus, where Walborn et al. (1999) found evidence for several generations of triggered star formation. Does our age-dating of clusters in the Antennae provide similar evidence for triggered star cluster formation? Figure 2 shows the pattern of ages in Knot $\mathrm{S}$ of the Antennae, the $\mathrm{H} \alpha$ image of Knot S, and a plot of the ages vs. the distance from the center of Knot $\mathrm{S}$.

Restricting the range to the inner 50 pixels $(\approx 140 \mathrm{pc})$ provides tentative evidence for some triggering, with a set of five older clusters found near the center of Knot S, and intermediate and young clusters found further out. Note the good correlation between the positions of the youngest clusters (squares in Figure 2a) and the positions of strong $\mathrm{H} \alpha$ flux image (Figure 2b). The $\mathrm{H} \alpha$ shell has apparently met with less resistance traveling to the right side of the image, where it has expanded to larger distance and fewer clusters have been formed. However, we find that the total mass of all the intermediate-age clusters is only about $5-10 \%$ of the mass of the central five clusters, hence triggered star formation is a relatively minor effect in this region. Several other knots of star clusters in the Antennae show similar patterns with relatively small fractions of mass being produced in triggered regions of star formation. Perhaps this indicates that most of the gas in the area was used in the original burst of star formation.

However, Knot B (Figure 3) presents both a simpler and a much more dramatic example of triggered star formation. In this case we find a single cluster with an age greater than $10 \mathrm{Myr}$ (and mass $\approx 10^{6} \mathrm{M}_{\odot}$ ), a smooth gradient in the cluster ages, and a population of younger clusters with a total mass $4 \times 10^{6} \mathrm{M}_{\odot}$, FOUR times greater than the cluster that appears to have triggered their formation. Triggering is not always weak! We note that the $\mathrm{H} \alpha$ supershell is centered on the older cluster, and the young clusters are located where the shell intersects the edge of a major dust lane. This implicates the older 

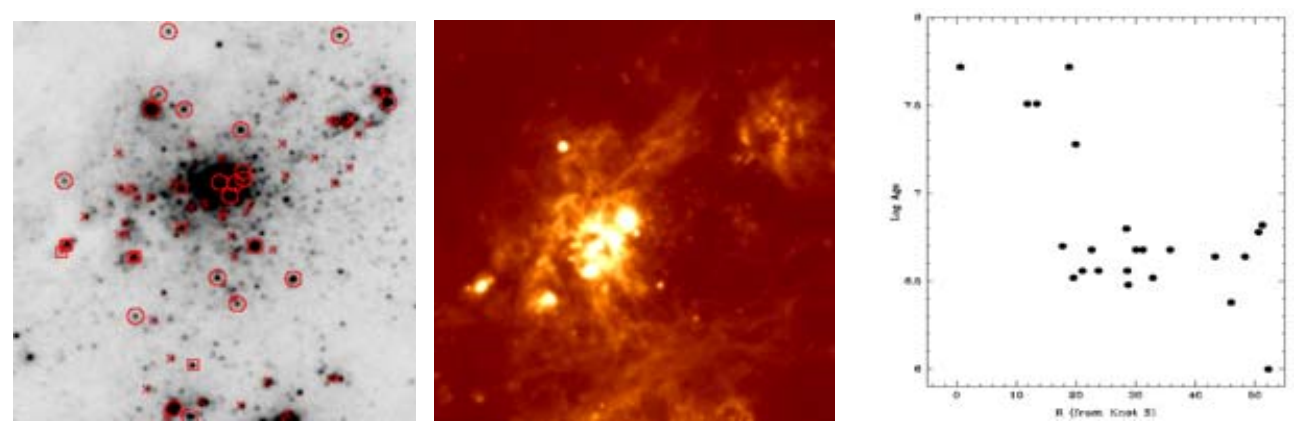

Figure 2. Left figure - I band images of Knot S in the Antennae with older ( $>10$ Myr: circles),

intermediate-aged ( 3 - $10 \mathrm{Myr}$, crosses), and very young (<3 Myr, squares) clusters. Center figure $-\mathrm{H}_{\alpha}$ image. Right figure - Age profile from center of Knot S. See Whitmore (2007) for a larger image.

cluster as the original spark. An analogy might be that if you throw a match down on a field of dry grass you might only get a few minor brushfires, but if you throw it down next to a tinder box something more impressive is formed.

\section{Differentiating stars from clusters}

While this might sound like a relatively unimportant technical issue, it is fundamentally important for answering the following two questions. What fraction of stars are formed in clusters vs. the field (i.e., does triggering primarily happen in isolation or in a clustered environment)? Is the initial cluster mass function a power law or a Gaussian (see Fall and Zhang 2001, de Grijs et al. 2005, Whitmore, Chandar, \& Fall 2006 for background)?

In Whitmore et al. (1999), differentiating stars from clusters using the undersampled WFPC2 image was one of our primary difficulties, leading us to conclude that the number of young star clusters in the Antennae was between 800 and 8000; a pretty big range! Our new ACS data provides a better opportunity for making this determination, and for studying the stars in their own right.

Figure 4 shows U-B vs V-I diagrams for 4 luminosity ranges of point-like objects around Knot S in the Antennae. The solid lines are Bruzual \& Charlot (2003) solar metalicity models for clusters. The dashed lines in the bottom left panel are Padova models for young stars. The dotted line shows a reddening vector, placed so that the objects above the line are primarily young clusters while the objects below the line are massive stars. Note that this only works when the objects are younger than about $50 \mathrm{Myr}$, at which point the Bruzual-Charlot models fall below the dotted line. Open squares are objects with PSFs that are indistinguishable from stars while filled circles appear to be slightly resolved (i.e., candidate clusters). The fact that all of the objects in the lower left panel (objects brighter than $\mathrm{M}_{V}=-10$ ) are in "cluster-space" is reassuring, since the brightest stars are believed to be approximately $\mathrm{M}_{V}=-9$ or fainter. Three candidate stars are found in the next luminosity bin (note that all three have $\mathrm{M}_{V} \approx 9.1$ ), but most of the other objects still appear to be clusters. As we progress to fainter bins, we find more candidate stars, but the majority of objects still appear to be clusters, based both on their spatial profiles and on their colors. In fact, a combination of size and colors appear to be the best discriminant between stars and clusters (see Whitmore et al. 2007 for a 

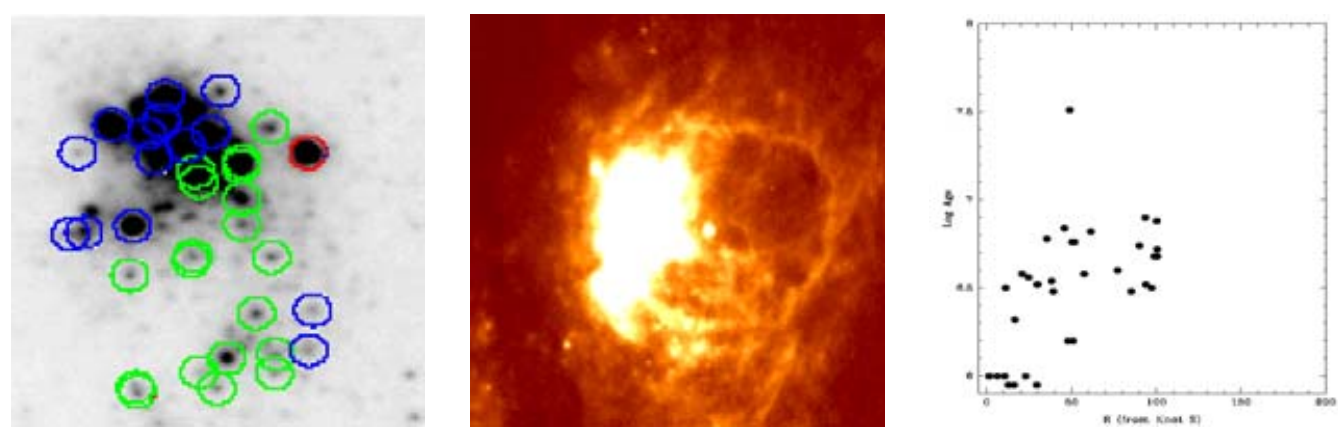

Figure 3. Same as Figure 2, except for Knot B. The center for the profile (right panel) is the set of bright young clusters on the left of the image, rather than the single older cluster in the upper right of Figure 3a. This older cluster is in the middle of the $\mathrm{H}_{\alpha}$ shell, implicating it as the original triggering cluster.

more detailed discussion), since some of the clusters appear to be so compact that they are indistinguishable from stars, even with ACS.

These results provide further evidence that most stars are indeed formed in clusters, and also show that the initial cluster mass function cannot have been a Gaussian, since this would require essentially all of the faint objects to be stars, rather than clusters.

\section{A general framework for understanding the demographics of star clusters}

Roughly 40 gas-rich mergers have now been observed by the Hubble Space Telescope. All of these show some number of young compact star clusters, similar to what we find in the Antennae. In addition, various authors have found young compact clusters in a variety of other galaxies, including starburst dwarf galaxies, barred galaxies, normal spiral galaxies, and the LMC. In general, the clusters found in non-interacting galaxies have properties similar to those seen in the mergers, but always fewer in number and generally fainter in luminosity. A reasonable hypothesis might therefore be that the most massive super star clusters can only be produced in violent environments rather than relatively quiescent environments such as those found in spiral galaxies? Indeed, various authors have suggested that there are two modes of star formation.

However, Whitmore (2003, originally presented in 2000 as astro-ph/0012546) and Larsen (2002) have shown that there is a smooth, continuous correlation between the brightest cluster in a galaxy and the number of clusters in the galaxy. This suggests that mergers and starburst galaxies may have the brightest clusters only because they have the most clusters. There may be a universal luminosity function with the correlation simply being due to statistics, rather than any special physics. This has been dubbed the "size-of-sample" effect, and is been included in a number of recent papers (e.g., Hunter et al. 2003). The number of clusters in mergers may be larger because conditions for triggering cluster formation are globally present in mergers but only locally present in spirals (e.g., along spiral arms).

There is growing evidence that the disruption rate of clusters may also be universal. For example, Fall, Chandar \& Whitmore (2005) find that $90 \%$ of the young clusters in the Antennae are disrupted each factor of 10 of time. This is also true in the Milky Way (Lada \& Lada 2003), and the SMC (Rafelski \& Zaritsky 2005, Chandar, Fall \& Whitmore 

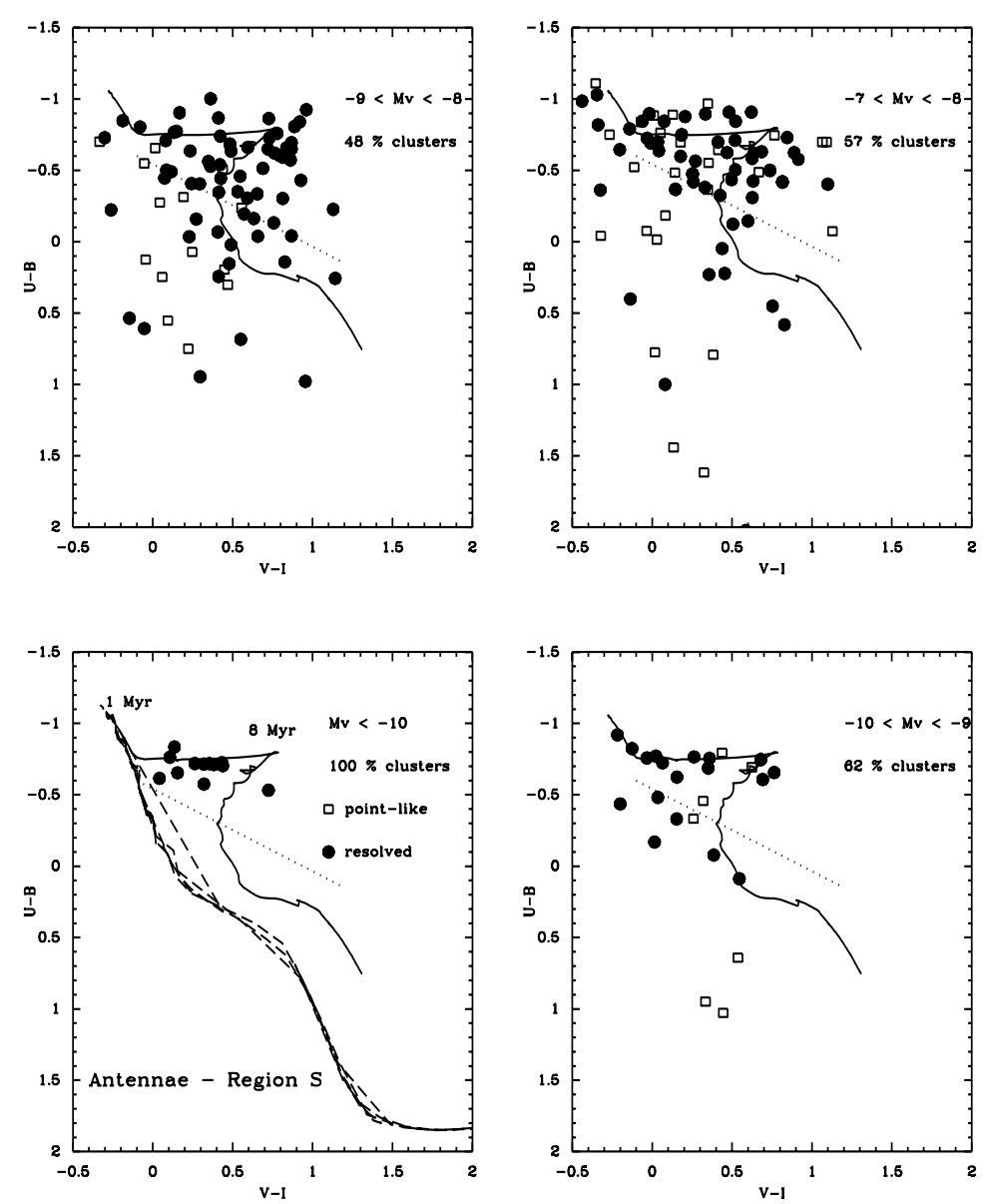

Figure 4. $U-B$ vs. $V-I$ color-color diagram for four luminosity ranges for a sample of objects around Knot S in the Antennae (from Whitmore et al. 2006; see text for details).

2006), as shown in Figure 5. Hence, the disruption rate appears to be a power law with index -1 .

These two results motivated us to develop a general framework for understanding the demographics of both star clusters and field stars, which we assume are formed as a by-product of the disrupted clusters (Whitmore, Chandar, Fall 2006). The ingredients for the model are: 1) a universal initial mass function (power law, index -2), 2) various star(cluster) formation histories that can be coadded (e.g., constant, Gaussian burst, ...), 3) various cluster disruption mechanisms (e.g., power law, index -1 for $<100 \mathrm{Myr}-$ infant mortality; constant mass loss for $>100 \mathrm{Myr}-2$-body relaxation), 4) convolution with observational artifacts and selection effects.

This simple model allows us to predict a wide variety of properties for the clusters, field stars, and integrated properties of a galaxy. Of particular relevance for the present paper is the fact that the model we have developed for the Antennae using this framework predicts that $\approx 8 \%$ of the UV luminosity should be from clusters rather than field stars, in agreement with the observations $(\approx 10 \%)$. Hence, our data is consistent with the idea 


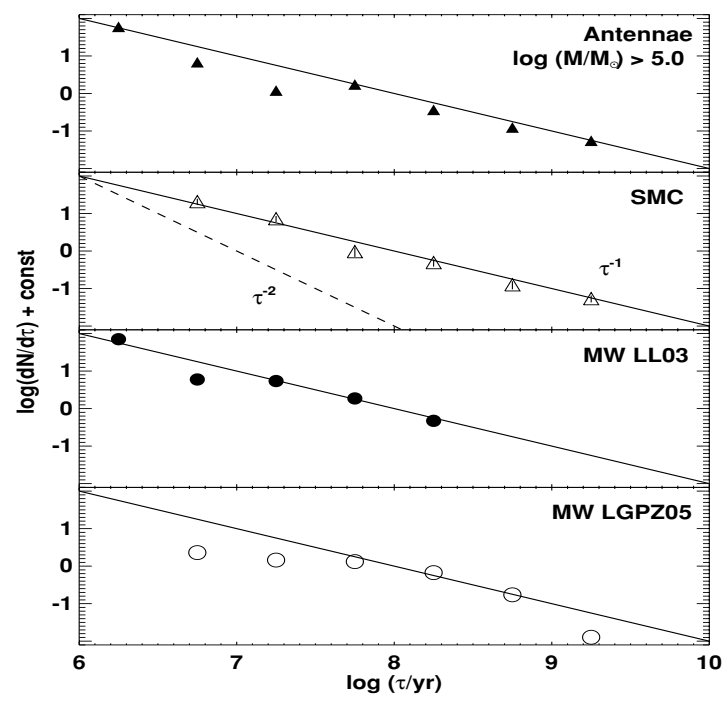

Figure 5. The age distributions for clusters in the Antennae (filled triangles), the SMC (open triangles), and the Milky Way filled and open circles. In each distribution we find that the number of clusters declines as $\tau^{-1}$; i.e., infant mortality $-90 \%$ loss each factor of 10 in time). See Whitmore, Chandar, \& Fall (2006) for details.

that all stars are born in clusters. Fall, Chandar \& Whitmore (2005) reach a similar conclusion based on $\mathrm{H} \alpha$ flux.

\section{Acknowledgements}

The authors wishes to acknowledge various coauthors on various projects discussed in this paper. These include Rupali Chandar, Francois Schweizer, Mike Fall, Qing Zhang, and Barry Rothberg.

\section{References}

Bruzual A. G. \& Charlot, S. 2003, MNRAS 344, 1000

Chandar, R., Fall, S. M. \& Whitmore, B. C. 2006, in press

de Grijs, R., Parmentier, G. \& Lamers, H. J. G. L. M. 2005, MNRAS 364, 1054

Fall, S. M., Chandar, R. \& Whitmore, B. C. 2005, ApJ 631, L133

Fall, S. M. \& Zhang, Q. 2001, ApJ 561, 751

Hunter, D. A., Elmegreen, B. G., Dupuy, T. T. \& Mortonson, M. 2003, AJ 126, 1836

Jog, C. \& Solomon, P. M. 1992, ApJ 387, 152

Kumai, Y., Hashi, Y. \& Fujimoto M. 1993, ApJ 416, 576

Lada, C. J. \& Lada, E. A. 2003, ARAA 41, 57

Larsen, S. S. 2002, $A J$ 124, 1393

Rafelski, M. \& Zaritsky, D. 2005, AJ 129, 2701

Toomre, A. 1977, in: B. M. Tinsley \& R. B. Larson (eds.) The Evolution of Galaxies and Stellar Populations (Yale: New Haven), p. 401

Walborn, N. R., Barba, R., H., Brandner, W., Rubio, M., Grebel, E. \& Probst, R. 1999, AJ 117,225

Whitmore, B. C. 2003, in: M. Livio, K. Noll, \& M. Stiavelli (eds.), A Decade of HST Science (Cambridge:Cambridge University), p. 153

Whitmore, B. C. 2007, in: M. Livio (ed.), Massive Stars: From Pop III and GRBs to the Milky Way (Cambridge:Cambridge University), in press 
Whitmore, B. C., Chandar, R. \& Fall, S. M. 2006, $A J$ in press

Whitmore, B. C., Gilmore, D., Leitherer, C., Fall, S. M., Chandar, R., Blair, W. P., Schweizer, F., Zhang, Q. \& Miller, B. W. 2005, AJ 130, 2104

Whitmore, B. C., Zhang, Q., Leitherer, C., Fall, S. M., Schweizer, F. \& Miller, B. W. 1999, AJ 118,1551

Zhang, Q., Fall, M. \& Whitmore, B. C. 2001, ApJ 561, 727

\section{Discussion}

DE GRIJS: (1) I'm a little worried about your distinction between stars and clusters if you're really working at the resolution limit. In a recent paper by Bastian, Lamers, etc., we showed that you need to use the full SED in addition to size estimates. Then all stars fall into the youngest age bin $(<6 \mathrm{Myr})$. Have you tried to assess your objects this way? (2) I think there is a subtle difference between your argument that the initial cluster luminosity function might be a power law and recent published results advocating lognormal initial CLFs. In your case, you include all objects, in the lognormal case one deals with the bound fraction only, so you would need to include the effects of infant mortality quantitatively (which is hard!) before making definitive statements... I think, therefore, that our results are not at odds with each other.

Whitmore: (1) We agree that using size alone is only partially successful in separating stars and clusters, but took some pains during the talk to show that in many cases it is actually quite easy using the new ACS data. However, we also use the spectral color information (see figure in paper) to help make the distinction. (2) Of course we would all like to know which clusters are bound and which are not. At present, this is impossible to do for all but the brightest clusters (using stellar velocity dispersions) directly). In your case, you use older clusters which are more likely to be bound, but then you have not direct information about the initial cluster mass function, and must make secondary arguments to try to infer the initial mass function. We prefer to work with all the clusters, whether they are bound or not, so that we can directly determine the initial mass function. We then try to estimate the fraction of unbound clusters, which causes the high infant mortality rate.

WALBORN: This is very important work, bridging the very local objects such as in the Magellanic Clouds where we have spectral classifications for all the OB stars to establish ages, and much more distant objects with no spatial resolution. The asymmetry of the triggering is remarkable and agrees with the local archetypes 30 Doradus, N11, NGC 3603 , and others, in which the second generation is always in one hemisphere relative to the first. This must be telling us something essential, perhaps that the initial cluster forms near an edge of the GMC in response to an external event, as opposed to a central collapse. As I'm sure you do, one must always bear in mind that a point source is not necessarily a single star. At this distance, 0.1 arcsec is about $10 \mathrm{pc}$.

Whitmore: Yes, I agree with everything you say. In particular your point that sometimes a cluster can be so concentrated that it can be indistinguishable from a star at this distance. This is an important sidelight of the color-color diagrams that I showed, that $10-20 \%$ of the objects in "cluster-space" appear to be unresolved.

MELNICK: 1. I question the idea that all stars are formed in clusters since in our galaxy up to $70 \%$ of the massive stars are not in clusters and are not runaway stars. 2. Don't 
you find it surprising that the (initial) mass function of clusters has the same slope in the antennae, in the LMC, in the Milky Way, and maybe everywhere else (i.e., is universal)?

Whitmore: 1. All we can really say is that most of the stars appear to form in groups and clusters. We certainly can't say that $100 \%$ do. 2 . This just shows that the process that makes clusters is probably pretty universal. I guess Nature has been kind to us.

ZINNECKER: From these observations of the Antennae and other colliding galaxies, what can you infer about the very process of triggering?

Whitmore: The specific thing we can say is that high speed (50-100 $\left.\mathrm{km} \mathrm{s}^{-1}\right)$ cloudcloud collisions are not directly responsible for triggering star and star cluster formation. The fact that the velocity dispersion between clusters is quite low $\left(<10 \mathrm{~km} \mathrm{~s}^{-1}\right)$, and roughly the same as the disk in a normal spiral disk, suggests to me that pre-existing GMCs are triggered into formation by an increase in ISM pressure, without changing the velocity distribution. An alternative possibility is a turbulent cascade of velocities for orbital collision speeds (hundreds of $\mathrm{km} \mathrm{s}^{-1}$ ) all the way down to disk-like velocity dispersions.

Alves: Just a comment to your result on the mass function of clusters. We find essentially the same mass spectrum for GMCs in both Can A and M83, which ties beautifully to your spectrum of clusters. the fact that you do not see a variation in the cluster mass spectrum between starburst galaxies and more star formation quiet galaxies also suggests a connection to the same underlying mass spectrum.

OEY: In response to Jorge Melnick's comment about field OB stars, we can take the power law mass function that you showed to estimate the fraction of field massive stars by extrapolating to individual stars (Oey, King \& Parker 204). But my question to you is: How can we distinguish between cluster dissolution and a recent/on-going burst of star formation? It seems most of the examples you showed are candidates for the latter.

Whitmore: For a particular galaxy, it is possible that we are catching a galaxy during a recent burst in the last $<10 \mathrm{Myr}$. However, when nearly everyone finds mean ages for young clusters in any galaxies with ongoing star formation (eg., most spirals) to be $<10 \mathrm{Myr}$, you have to start wondering. Infant mortality (dissolution of $90 \%$ of the clusters each decade of log time) is such a dominant effect that it results in mean cluster ages $<10 \mathrm{Myr}$ even if the star formation is not very constant. Another way to demonstrate how dominant infant mortality is is to break the Antennae into 4 quadrants. It is impossible to coordinate a starburst over the entire galaxy, and in fact there is good evidence that the overlap region is currently where the most recent star formation is. However , the $\mathrm{dN} / \mathrm{dT}$ diagram looks very similar in all 4 quadrants, but the mean age is always $<10 \mathrm{Myr}$, even in regions which had a strong burst $\sim 100 \mathrm{Myr}$ ago. I will include this figure in the proceedings. 